\title{
Viscoelastic Behaviour of Pumpkin Balloons
}

\author{
T. Gerngross, Y. Xu and S. Pellegrino* \\ Department of Engineering, University of Cambridge \\ Trumpington Street, Cambridge, CB2 1PZ, UK
}

\begin{abstract}
The lobes of the NASA ULDB pumpkin-shaped super-pressure balloons are made of a thin polymeric film that shows considerable time-dependent behaviour. A nonlinear viscoelastic model based on experimental measurements has been recently established for this film. This paper presents a simulation of the viscoelastic behaviour of ULDB balloons with the finite element software ABAQUS. First, the standard viscoelastic modelling capabilities available in ABAQUS are examined, but are found of limited accuracy even for the case of simple uniaxial creep tests on ULDB films. Then, a nonlinear viscoelastic constitutive model is implemented by means of a user-defined subroutine. This approach is verified by means of biaxial creep experiments on pressurized cylinders and is found to be accurate provided that the film anisotropy is also included in the model. A preliminary set of predictions for a single lobe of a ULDB is presented at the end of the paper. It indicates that timedependent effects in a balloon structure can lead to significant stress redistribution and large increases in the transverse strains in the lobes.
\end{abstract}

Key words: atmospheric balloons, viscoelasticity, creep, polyethylene film, Schapery

\section{Introduction}

The NASA Ultra Long Duration Balloon (ULDB) project is developing balloon structures with diameters of up to $120 \mathrm{~m}$. ULDB designs involve over 200 identical gores made of a thin polymeric film attached to high stiffness, meridional tendons. While the behaviour of the tendons is essentially timeindependent, the film shows considerable viscoelastic behaviour.

* Corresponding author.

Email address: sp28@cam.ac.uk (S. Pellegrino). 
Thin polymeric films have been used in atmospheric balloons for many years and their viscoelastic behaviour has been studied extensively. Of particular relevance to the present study is a description of the ULDB film material by Rand and co-workers (Henderson, Caldron and Rand, 1994; Rand, Grant and Strganac, 1996; Rand, Henderson and Grant, 1996) that is based on Schapery's classical work (Schapery, 1969). In this paper, this formulation will be denoted as the Schapery-Rand model. An alternative model, based on a decomposition into thermodynamically derived functions, instead of the master curve used in the Schapery model, has been proposed by Vialettes et al. (2005) in connection with CNES super-pressure balloons.

This paper uses a User-Defined Material (UMAT) subroutine in the commercial finite-element software ABAQUS to study the time variation of the strain and stress distribution in a pumpkin balloon. Detailed predictions from this approach are compared to photogrammetry measurements on simple balloons of cylindrical shape. Preliminary results for the behaviour of a $10 \mathrm{~m}$ diameter pumpkin balloon with 145 lobes are obtained, also with ABAQUS, using a simple power-law creep model. These results indicate that time-dependent effects in a balloon structure can lead to a significant stress redistribution and large increases in the transverse strains in the lobes.

The paper is set out as follows. First, in the next section, the Schapery-Rand model is outlined and the numerical parameters are defined for the case of the ULDB film. Next, in Section 3, the standard viscoelastic modelling capabilities available in ABAQUS are investigated for uniaxial test cases. It is shown that the built-in viscoelastic models give reasonable results, but not a fully accurate fit to experimental data. It is also found that analytical predictions from the Schapery-Rand model are much more accurate. Section 4 outlines a user-defined material subroutine in ABAQUS, whose performance is then tested for uniform biaxial creep in two cylinder tests. The numerical results are compared to experimental creep strain data. Finally, in Section 6, simple predictions of the creep strains and stresses in a pumpkin balloon, based on the ABAQUS power-law creep model are presented. Section 7 concludes the paper.

\section{Nonlinear Viscoelastic Model}

\subsection{Schapery-Rand Model}

Schapery (1969) proposed a nonlinear viscoelastic constitutive model where the transient material behavior is defined by a function known as the master curve. This is a plot of creep compliance, $D$, additional to the instantaneous 
compliance, $D_{0}$, in terms of a time variable $\psi$, known as the reduced time. Nonlinearities are captured by including four functions of stress and temperature. The dependence of reduced time on temperature is captured by an additional function, of temperature only.

More precisely, the reduced time is defined as

$$
\psi(t)=\int_{0}^{t} \frac{d \tau}{a_{T}(T) a_{\sigma}(T, \sigma)}
$$

where $a_{T}$ is a temperature dependent function and $a_{\sigma}$ a stress and temperature dependent function.

Then, the total uniaxial strain is obtained from:

$$
\varepsilon(t)=g_{0} D_{0} \sigma(t)+g_{1} \int_{0}^{t}\left(D(\psi(t)-\psi(\tau)) \frac{d\left(g_{2} \sigma(\tau)\right)}{d \tau} d \tau\right.
$$

where the first term represents the instantaneous, i.e. elastic response while the second term describes the transient response. Note the nonlinearity factors: $g_{0}$ is the change of instantaneous elastic compliance, $g_{1}$ is the change of transient compliance, and $g_{2}$ is the sensitivity to transient stress. They are all functions of stress and temperature.

Rand, Grant and Strganac (1996) extended Schapery's uniaxial formulation to the case of plane stress. The nonlinearity functions were related to a single effective stress $\bar{\sigma}$ given, at any time $t$, by

$$
\bar{\sigma}=\sqrt{\sigma_{1}^{2}+2 A_{12} \sigma_{1} \sigma_{2}+A_{22} \sigma_{2}^{2}+A_{66} \sigma_{12}^{2}}
$$

where $\sigma_{1}, \sigma_{2}$ are the normal stress components in the machine and transverse directions of the film, respectively, and $\sigma_{12}$ is the shear stress. The coefficients $A_{i j}$ are chosen such that the nonlinearity functions for the machine direction can be used to determine the nonlinearities in any direction.

\subsection{ULDB Film Parameters}

A material that has been used extensively for stratospheric balloons is a Linear Low Density Polyethylene (LLDPE) called StratoFilm 372, or SF372. Experimental data for this material has been published (Henderson, Caldron and Rand, 1994; Rand, Grant and Strganac, 1996; Rand, Henderson and Grant, 1996). More recently, a film called StratoFilm 420 has been introduced, which is a three layer extrusion from the same resin as SF372, with a total thickness of $0.038 \mathrm{~mm}$. The material used for the experiments presented in this paper was SF430; this is essentially the same as SF420, but without an additive for 


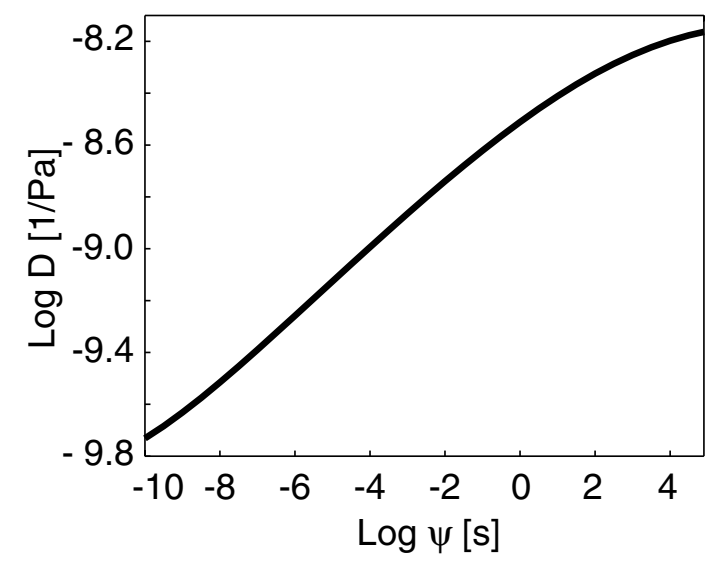

Fig. 1. Master curve for transient creep compliance of SF372 in machine direction, from Rand, Henderson and Grant (1996).

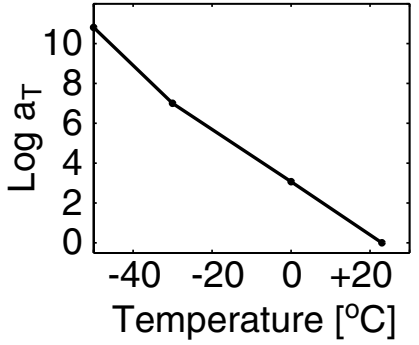

(a) $a_{T}$

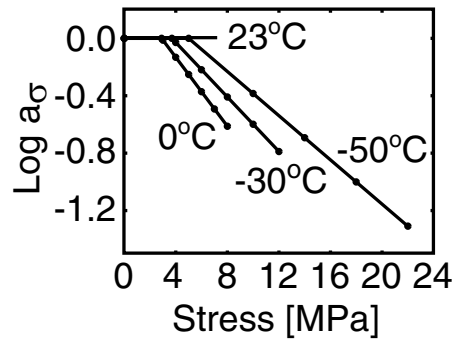

(b) $a_{\sigma}$

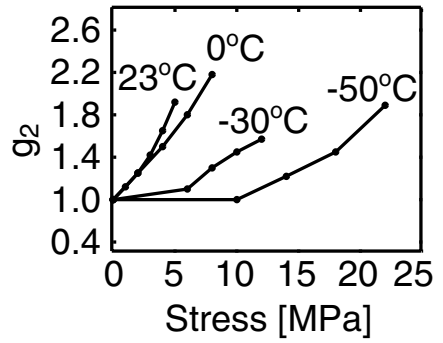

(c) $g_{2}$

Fig. 2. Nonlinearity functions for SF372, from Rand, Henderson and Grant (1996).

protection from ultraviolet light. After some initial uniaxial creep experiments on SF430, it was decided that it would be reasonable to use the material data for SF372.

Figures 1 and 2 show the viscoelastic material data in the machine direction for SF372. The data presented includes the master curve, the temperature shift function $a_{T}$, and the nonlinearity functions $a_{\sigma}$ and $g_{2}$. The nonlinearity parameters $g_{0}$ and $g_{1}$ in the general Schapery model, Equation (2), are set equal to 1.0. Note that the master curve relates to a temperature of $23^{\circ} \mathrm{C}$. The instantaneous elastic compliance $D_{0}=3 \times 10^{-10} \mathrm{~Pa}^{-1}$ has been estimated at the smallest $\psi$ available, $\psi \approx 10^{-10}$ s. The coefficients of Equation (3) are listed in Table 1 , for plane stress at $23^{\circ} \mathrm{C}$.

Table 1

Biaxial stress coefficients for SF372 at $23^{\circ} \mathrm{C}$

\begin{tabular}{|c|c|c|}
\hline$A_{12}$ & $A_{22}$ & $A_{66}$ \\
\hline-1.08 & 1.18 & 6.05 \\
\hline
\end{tabular}




\section{Viscoelastic Capabilities in ABAQUS}

\subsection{Power Law Creep Option}

The finite element package ABAQUS offers a power-law model that can be used in time hardening or strain hardening form, via the *creep function. Both forms define an equivalent uniaxial creep strain rate that depends on the equivalent uniaxial deviatoric stress, the total time, and three user-defined functions of temperature. The time hardening form is expressed as

$$
\dot{\bar{\varepsilon}}^{c r}=A \bar{\sigma}^{n} t^{m}
$$

Assuming constant uniaxial stress $\bar{\sigma}_{0}$ and constant temperature, integration of Equation (4) yields

$$
\bar{\varepsilon}^{c r}=\frac{A}{m+1} \bar{\sigma}_{0}^{n} t^{m+1}
$$

The parameters $A, n, m$ can be obtained by fitting Equation (5) to the LLDPE master curve, Figure 1, but this requires that one particular value of $\bar{\sigma}_{0}$ be assumed.

This approach is found to be quite accurate at or near the particular stress at which the model was fitted to the experimental data, however there can be significant errors at other stress levels. For example, Figure 3 shows (among other results) the power-law model predictions obtained for a uniaxial stress of $4 \mathrm{MPa}$ using a model fitted to experimental data at $\bar{\sigma}_{0}=2 \mathrm{MPa}$. The coefficients of the model are listed in Table 2.

Table 2

Coefficients for power-law fit $\left(T=20^{\circ} \mathrm{C}, \bar{\sigma}_{0}=2 \mathrm{MPa}\right)$

\begin{tabular}{|c|c|c|}
\hline$A$ & $n$ & $m$ \\
\hline $1.82 \times 10^{-4}$ & 1.63 & -0.925 \\
\hline
\end{tabular}

\subsection{Shear Test Data Option}

Another possibility is to use the $* v$ iscoelastic command in conjunction with $*$ shear test data, in order to generate a viscoelastic model based on experimental data from either creep or relaxation tests.

This approach is based on a stress relaxation formulation that assumes strain as the independent state variable. Similar to the way creep data was used in Equation (2), stress relaxation can be written as (McCrum, Buckley and 
Bucknall, 2003)

$$
\sigma=\int_{0}^{t} E(t-\tau) \frac{d \varepsilon(\tau)}{d \tau} d \tau
$$

where $E(t)$ is the tensile stress relaxation modulus at time $t$. The ABAQUS formulation uses normalised shear test data, and hence Equation (6) needs to be rewritten as (ABAQUS, 2003):

$$
\tau(t)=G_{0} \int_{0}^{t} g_{R}(t-\tau) \frac{d \gamma(\tau)}{d \tau} d \tau
$$

where $\gamma$ is the shear strain and $g_{R}(t)$ is the normalised shear relaxation modulus, defined as $g_{R}(t)=G_{R}(t) / G_{0}$. Here $G_{R}(t)$ is the time-dependent shear relaxation modulus and $G_{0}=G_{R}(0)$ is the instantaneous shear modulus.

The normalised relaxation modulus is defined by a sum of exponentials known as a Prony series, and can be written as:

$$
g_{R}(t)=1-\sum_{i=1}^{N} g_{i}\left(1-e^{-\frac{t}{\tau_{i}}}\right)
$$

ABAQUS will compute the terms in the Prony series automatically from a given set of normalized shear creep compliance data, which can be derived by adding the instantaneous compliance to the master curve data and dividing by the instantaneous compliance. The Poisson's ratio of the film is assumed to remain unchanged over time, and hence does not appear in the normalized shear compliance.

This method is a linear version of Schapery's constitutive equation, as can be seen by comparing Equation (2) with Equation (6). However, stress dependent nonlinearities are, again, not captured by this approach.

When the master curve in Figure 1 was entered as creep test data spanning 16 decades of time, ABAQUS encountered problems with the automatic conversion to a Prony series. To overcome this problem the creep data was transformed into relaxation data and then the automatic conversion to a Prony series worked fine.

\subsection{Validation}

Predictions from the two different viscoelastic options in ABAQUS were compared to a direct solution of the Schapery-Rand nonlinear viscoelastic model with the parameters defined in Section 2, and also to experimental measurements. As already mentioned, the parameters for the ABAQUS models were obtained by fitting to experimental data at a constant stress of $2 \mathrm{MPa}$. The 
accuracy of the models was tested by simulating the creep response of a uniaxial specimen subject to a constant stress of $2 \mathrm{MPa}$ and it was found that very good agreement could be achieved with the $*$ viscoelastic option, by modifying the slope of the function $g_{2}$ values for $23^{\circ} \mathrm{C}$, in Figure 2(c). The results obtained with the $*$ creep option were less good.

A validation exercise was then carried out for uniaxial creep tests at a constant stress level of $4 \mathrm{MPa}$, to assess the accuracy of stress-independent creep models for SF372. The results are shown in Figure 3.

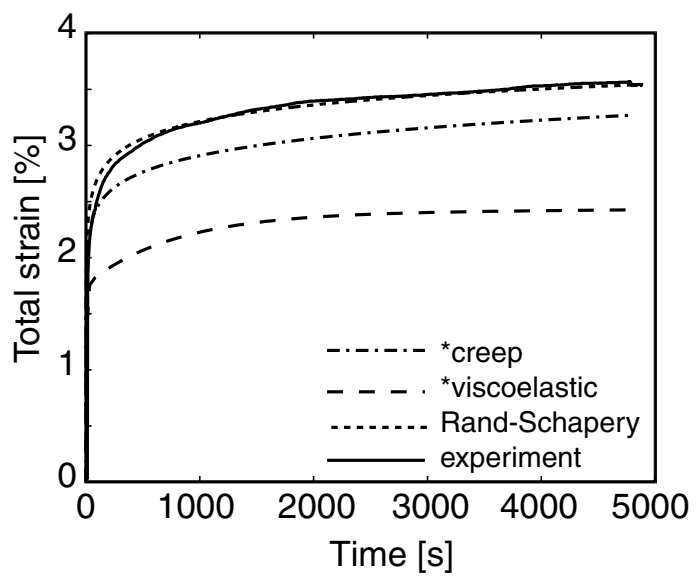

Fig. 3. Comparison of viscoelastic models at stress of $4 \mathrm{MPa}$.

Both ABAQUS options give rather poor results, the power-law model based on the $*$ creep option predicts strains up to $10 \%$ lower. The $*$ viscoelastic option, which follows the linear Schapery viscoelastic constitutive equation, but similarly neglects the stress dependent nonlinearities of the material, underpredicts the creep strains by up to $40 \%$.

On the other hand, the creep strains predicted by the Schapery-Rand model practically coincide with the experimental data. The next section describes an implementation of this model in ABAQUS in the form of a user defined material.

\section{ABAQUS UMAT Subroutine}

To implement the Schapery-Rand constitutive model in a numerical algorithm, Equation (2) needs to be rewritten in incremental form. A numerical integration method for a three-dimensional, isotropic, viscoelastic material has been presented by Haj-Ali and Muliana (2004). Following the same approach, during the present study the Schapery-Rand model was implemented 
in ABAQUS. The present work could be readily extended to any displacement based finite element software, where the strain components are used as independent state variables.

The viscoelastic properties are entered as material constants in the ABAQUS input file $*$ user material. The incremental formulation requires the transient compliance $D$ to be expressed in terms of a Prony series, as in Section 3.2. 16 terms were included.

A graphical depiction of the algorithm is shown in Figure 4. ABAQUS calls UMAT during each increment and for each integration point. The ABAQUS interface for a user defined material passes a time increment $\Delta t$ and a corresponding strain increment $\Delta \varepsilon^{A B A Q U S}$, this is determined using the Jacobian matrix computed at the end of the previous time increment.

Every time UMAT is called it starts with an estimation of a trial stress increment, $\Delta \sigma^{\text {trial }}$, based on the nonlinearity parameters at the end of the previous time increment. With this initial guess an iterative loop is entered, where integration of Equation (2) yields a trial strain increment, $\Delta \varepsilon^{\text {trial }}$, which is then compared with $\Delta \varepsilon^{A B A Q U S}$. If required, the trial stresses and the nonlinearity parameters are corrected and the loop is repeated. Alternatively, if the strain error residual is below a tolerance (set to tol $=10^{-7}$ ) UMAT exits the loop and updates the Jacobian matrix $\frac{\partial \Delta \sigma_{j}}{\partial \Delta \varepsilon_{i}}$ and the stresses $\sigma_{j}$. Also, at the end of the increment the history for every strain/stress component and every Prony term is stored using the statev array.

ABAQUS passes:

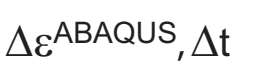
stress/strain history

$$
\Delta \varepsilon^{\text {trial }}=\varepsilon^{t}-\varepsilon^{t-\Delta t}
$$

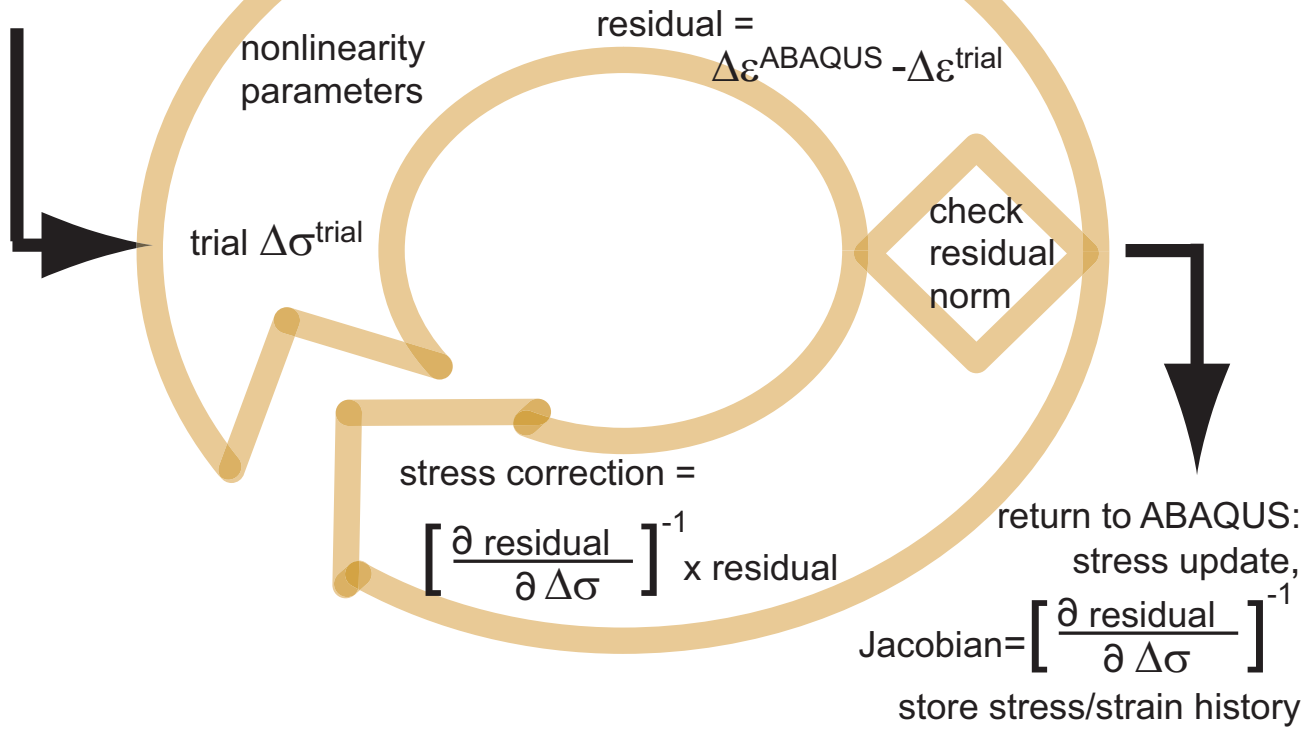

Fig. 4. Iterative algorithm in UMAT. 


\section{Verification of UMAT Subroutine}

The accuracy of the nonlinear viscoelastic model implemented in the ABAQUS UMAT subroutine was verified against experimental creep strain measurements on two pressurized cylinders.

\subsection{Experiment}

The cylinders had a diameter of $300 \mathrm{~mm}$ and height of $760 \mathrm{~mm}$, and were made of SF430. The overall test layout can be seen in Figure 5(a).

\subsubsection{Specimen Preparation and Setup}

Two layers of SF430 were placed on top of each other and heat-sealed with a soldering iron. Symmetry was achieved by forming two diametrically opposite, parallel seams. End-fittings made of MDF panels were attached to the cylinder with jubilee clamps and sealed with silicone and felt padding.

At mid-height the cylinder was fitted with nine self-adhesive coded targets for photogrammetry strain measurements, plus 2 targets for reference. The targets were located over a $40 \times 40 \mathrm{~mm}$ square region, see Figure 5 (b).

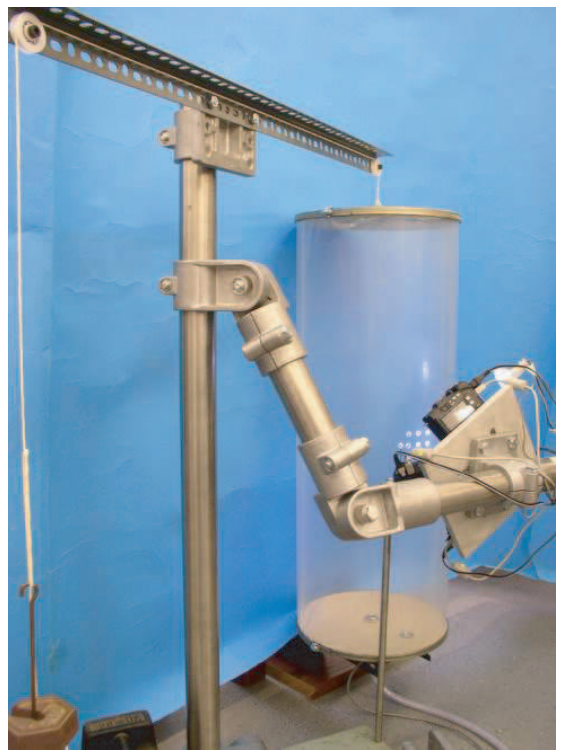

(a) Experimental setup

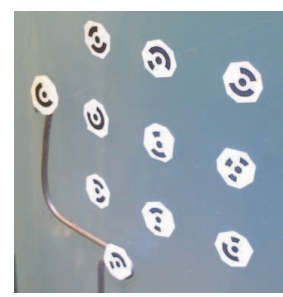

(b) Measurement region

Fig. 5. Cylinder specimen.

The bottom end fitting was clamped to a rigid support, while the top end 
fitting was suspended from a string going over a pulley arrangement to a counterweight hanger, see Figure 5(a). The cylinder was attached to an airline for pressurization and to a Sensor Technics pressure transducer with a maximum pressure of $2500 \mathrm{~Pa}$.

Four Olympus SP-350 digital cameras (with a resolution of 8.0 Mpixel) were connected to a personal computer and mounted in front of the target area. The cameras were triggered via the computer and so produced sets of four simultaneous photos of the coded targets, each from a different view point.

\subsubsection{Procedure}

Initially, the weights on the counterweight hanger were set to match the weight of the top end fitting, in order to remove any load from the cylinder.

The pressure regulator was set to the nominal experimental pressure, see Table 3. At the same time the axial load on the cylinder was increased by hanging additional weights through the counterweight hanger. The cylinder pressure was recorded throughout the experiment. While the increase of the counterweight resulted in an instantaneous increment in axial loading, full pressurization was reached only after $\sim 10$ seconds.

Under loading the viscoelastic film deformed and the movement of the coded targets was recorded by taking a series of close-up photos. Photos of the measurement area were taken, initially at intervals of a few seconds and increasing to five minutes towards the end of the experiment. Three-dimensional coordinates of the targets were then computed by means of the photogrammetry software Photomodeler (2004). The strain components in the axial and hoop directions were computed from the changes of coordinates of the targets over time.

\subsection{Analysis}

It was assumed that the cylinder would be under a biaxial state of stress throughout the experiment, and so wrinkle free. This allowed the film to be modelled with triangular membrane elements; a mesh of 72 M3D3 elements in the hoop direction and 56 elements in the longitudinal direction was used. Wrinkling of the film, which occurs near the end-fittings, was neglected because the creep measurements were taken away from the ends. One end fitting was

fully constrained, whereas the other end of the cylinder was allowed to move axially.

A uniform internal pressure was applied, through the $*$ dload command, and 
axial forces were simultaneously applied through the $*$ cload command. Both loads were increased from zero to their final value in a single instantaneous step and kept constant for $5100 \mathrm{~s}$.

The film was assumed to be isotropic with a Poisson's ratio of 0.49 and a constant thickness of $0.038 \mathrm{~mm}$. The analysis was run at a constant room temperature of $23^{\circ} \mathrm{C}$. Figure 6 shows the axial and hoop strains at $5100 \mathrm{~s}$, in cylinder 1.
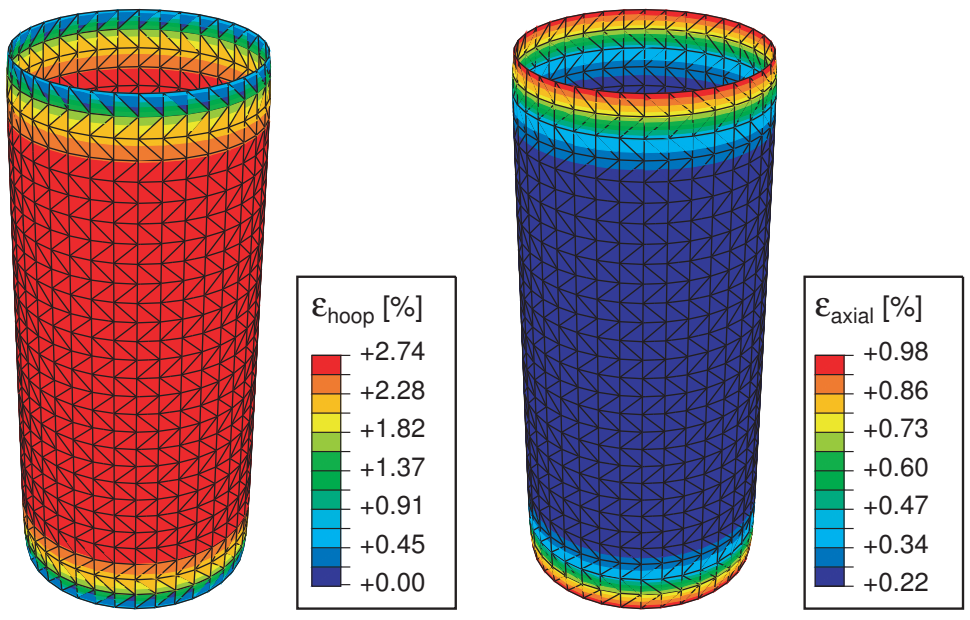

Fig. 6. Strain distribution after $5100 \mathrm{~s}$.

\subsection{Comparison}

Two cylinders were tested at different pressures and with different axial loads, see Table 3. Both cylinders were built with the material's machine direction aligned with the cylinder's hoop direction.

Table 3

Cylinder experiments

\begin{tabular}{|c|c|c|}
\hline Cylinder & Pressure $[\mathrm{Pa}]$ & Axial load $[\mathrm{N}]$ \\
\hline 1 & 930 & 6.28 \\
\hline 2 & 1100 & 22.22 \\
\hline
\end{tabular}

The total strains, i.e. including both instantaneous and time-dependent response, from experiment and analysis are compared in Figure 7. The results from the analysis based on the isotropic UMAT, with an assumed Poisson's ratio of 0.49 , show a significant difference from the experimental measurements. Remarkably, the strain in the axial direction decreases over time in the isotropic model. This is due to anisotropy that cannot be captured with the isotropic UMAT. 
An anisotropic version of the UMAT subroutine (which will be presented in detail elsewhere) has been implemented to establish the accuracy of a more refined material model. In the anisotropic UMAT the hoop compliance, which is in the machine direction of the film, was left unchanged but the longitudinal compliance was multiplied by a factor of 0.72 . Also, the Poisson's ratios were $\nu_{12}=0.49$ and $\nu_{21}=0.68$.

The dotted lines in Figures $7(\mathrm{a})$ and $7(\mathrm{~b})$ show the strains predicted by the anisotropic model; these predictions agree well with the measurements.

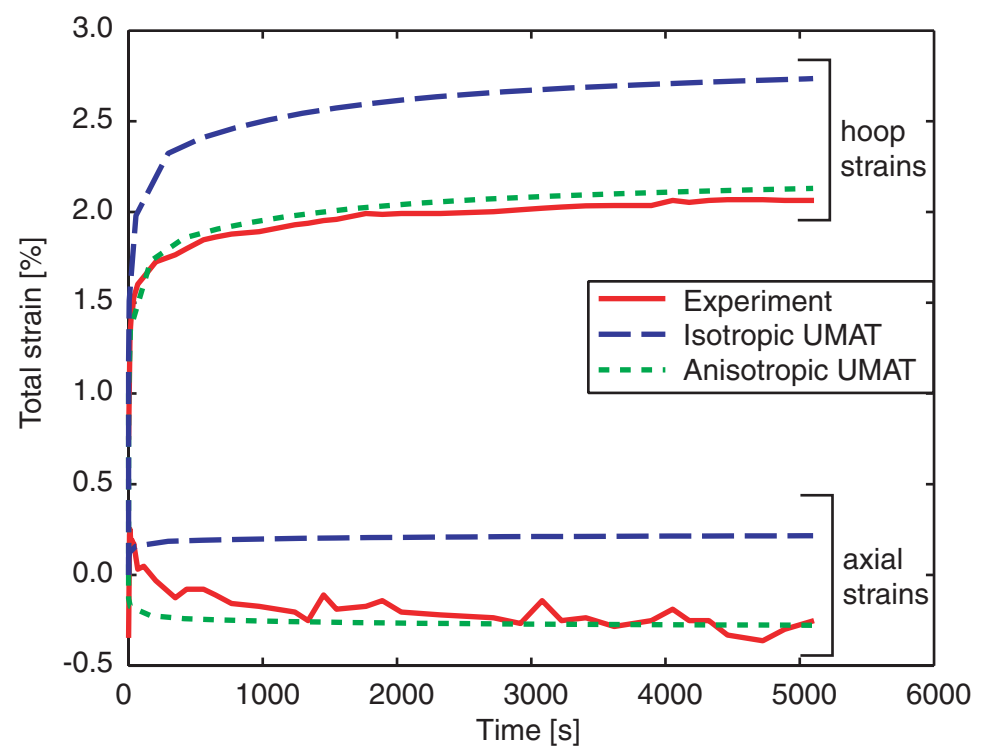

(a) Pressure $930 \mathrm{~Pa}$, axial load $6.28 \mathrm{~N}$

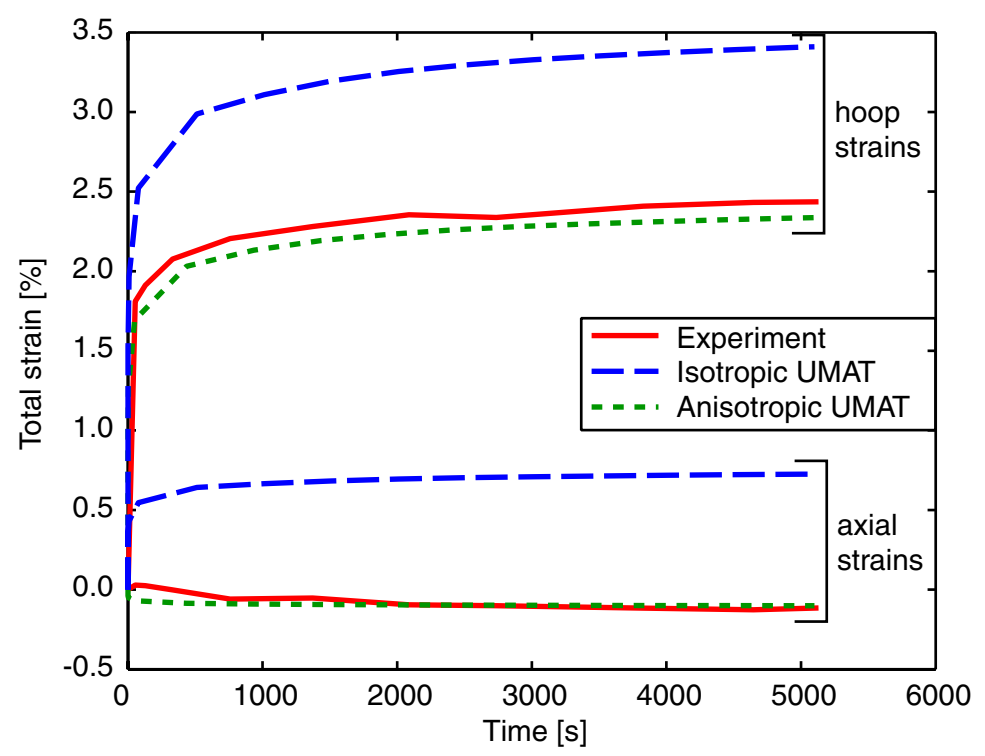

(b) Pressure $1100 \mathrm{~Pa}$, axial load $22.22 \mathrm{~N}$

Fig. 7. Comparison of total strains in two cylinders. 


\section{Preliminary Study of Balloon Behaviour}

A preliminary viscoelastic simulation of a pumpkin balloon with a diameter of $10 \mathrm{~m}$ and consisting of 145 lobes has been carried out. In the simulation the pressure was instantaneously increased to $1200 \mathrm{~Pa}$ at $t=0$ and then kept constant.

The lobe cutting pattern was based on the constant radius design described by Smith and Rainwater (2004) and shown in Figure 8. The width, $d$, of the cutting pattern was defined by the corresponding value of the cord length, $c$, obtained from the overall shape of the balloon (isotensoid) - and by assuming that the radius of curvature of the inflated lobe is $r=0.126 \mathrm{~m}$ everywhere. See Pagitz and Pellegrino (2007) for details on the modelling technique.

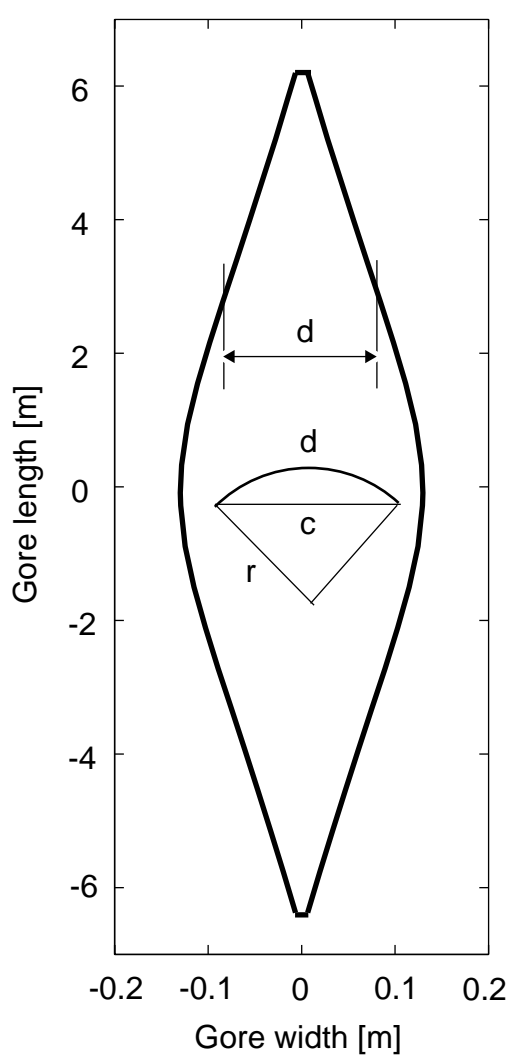

Fig. 8. Gore cutting pattern, the tips are removed for the end caps.

The properties of SF372 were used for the simulation (isotropic, instantaneous modulus $3333 \mathrm{MPa}$, Poisson's ratio 0.566, thickness $0.038 \mathrm{~mm}$ ). The ABAQUS built-in power-law model discussed in Section 3.1 was used. The coefficients for the time-hardening formulation were fitted to the SF372 experimental data at a stress $\bar{\sigma}_{0}$ in the range 4 to $6 \mathrm{MPa}$ and assuming a temperature of $20^{\circ}$, their values are given in Table 4 . The tendons stiffness was set to $170 \mathrm{kN}$ and a time-independent behaviour was assumed. 
Table 4

Coefficients for power-law fit $\left(T=20^{\circ} \mathrm{C}\right)$

\begin{tabular}{|c|c|c|}
\hline$A$ & $n$ & $m$ \\
\hline $1.27 \times 10^{-4}$ & 1.63 & -0.94 \\
\hline
\end{tabular}

The variation of strains and stresses over 1000 minutes is plotted in Figure 9. The initial strains are smaller than $0.2 \%$ both in the meridional direction and in the hoop direction. Over time, the meridional strains remain essentially unchanged in most of the lobe, apart from the equatorial region where they increase six times, whereas the hoop strains increase by a factor of about six in most of the lobe, apart from the equatorial region where they remain approximately constant. The initial stress distribution has peak values of approximately $8 \mathrm{MPa}$, quickly decreasing to about half this value and then remaining approximately constant.

A simple pseudo-elastic approximation is sometimes used to predict viscoelastic stress distributions and for the present case Rand has estimated a pseudoelastic modulus of $138.7 \mathrm{MPa}$. Figure 10 presents a comparison of the stress distribution that is computed from the power-law viscoelastic model, with that obtained from the simplified linear model. It can be seen that the linear model produces a roughly correct estimate of the hoop stresses, but underestimates the peak meridional stress.

\section{Discussion and Conclusion}

This paper has shown the importance of viscoelastic effects in the behaviour of balloon structures made of LLDPE. In the particular case of ULDB balloons our preliminary analysis results have shown that the stress distribution tends to become more uniformly distributed, over time. This potentially important result will need to be verified by a more detailed analysis, of course, and temperature effects should also be considered.

We have shown that the biaxial behaviour of the ULDB film material SF372 is well modelled by the Schapery-Rand non-linear, anisotropic viscoelastic model. This behaviour has been captured by the anisotropic version of our ABAQUS UMAT subroutine, as shown by comparing numerical predictions to experimental measurements taken on two cylinders. We have found that the isotropic version of the model is of limited accuracy. We have also found that the standard power-law creep and viscoelastic models available in ABAQUS are less accurate than the Schapery-Rand models, even in the case of problems where the stress distribution is relatively uniform and it is possible to determine the best-fit model parameters near the stress level of interest. 


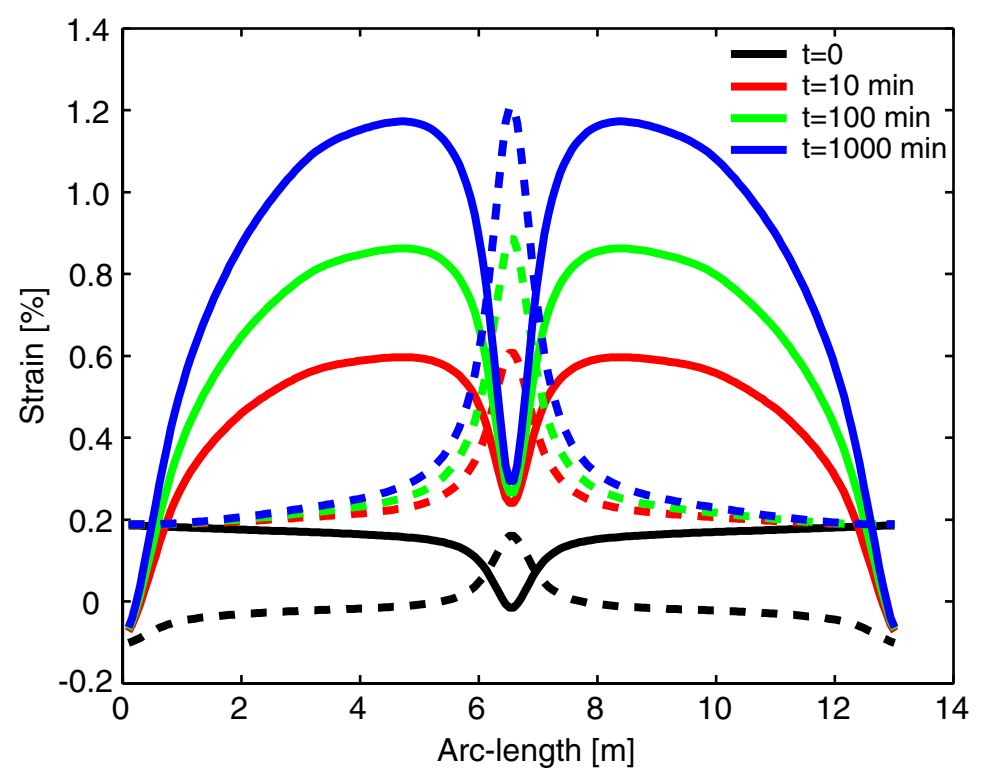

(a) Strain distribution

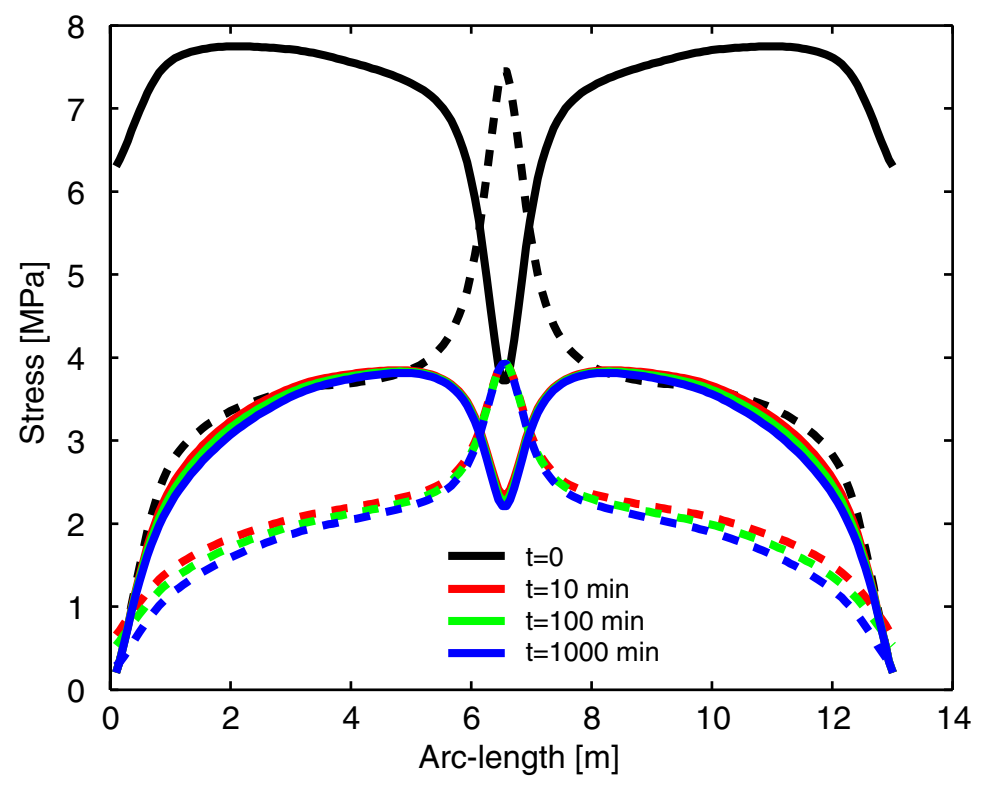

(b) Stress distribution

Fig. 9. Distribution of total strains and stresses along the centerline of a pumpkin balloon lobe; solid lines denote hoop strains/stresses, dotted lines denote meridional strains/stresses.

The ULDB film is a blown film that can be expected to show anisotropic behaviour. This became apparent in Section 5.3 when numerical and experimental results were compared. While under a biaxial state of stress the higher stiffness of the material in the axial direction resulted in lower strains compared to the isotropic case, the axial strains were actually observed to be decreasing. This behaviour cannot be captured by the isotropic model. 


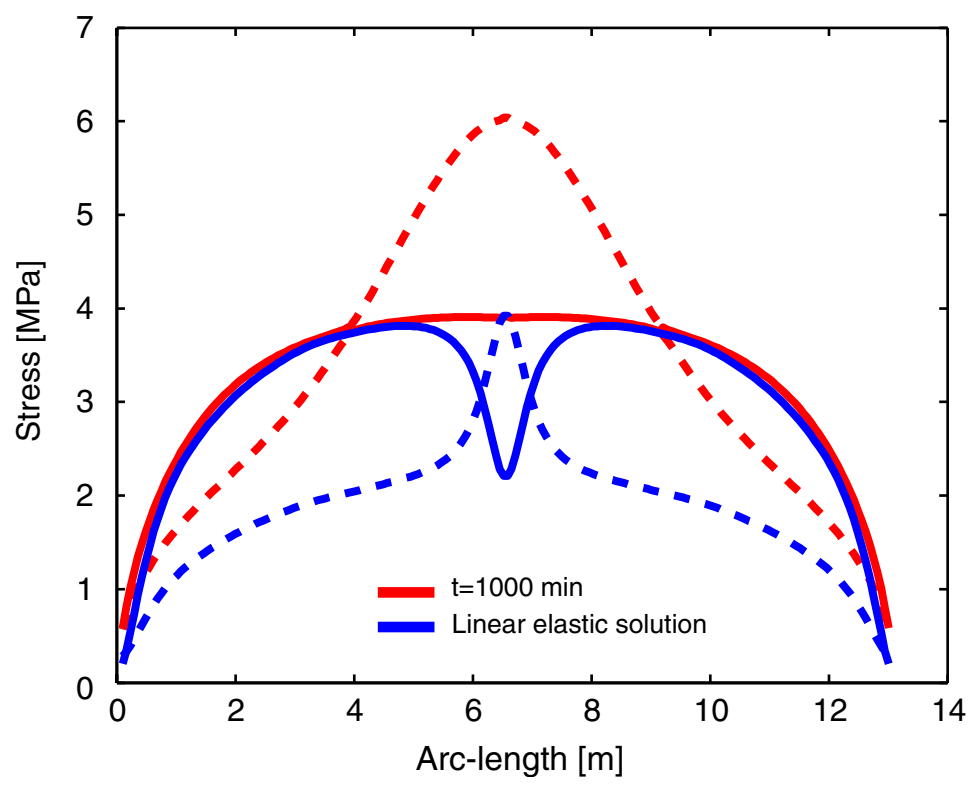

Fig. 10. Comparison of stress distribution obtained from pseudo-elastic simulation with viscoelastic stress distribution after $1000 \mathrm{~min}$; solid lines denote hoop stresses, dotted lines denote meridional stresses.

The discrepancy between the initial behaviour observed experimentally and the anisotropic model predictions may be due to the rate of application of the two components of the loading, at the beginning of the experiment. The axial load was applied very quickly, while the nominal pressure was reached only after about 10 seconds. Furthermore, a general reason for these discrepancies might be the use of material data describing SF372 in the analysis, while the material that was used with the experiment is the current ULDB material SF430.

\section{Acknowledgments}

We thank Dr Jim Rand for advice and providing film material data to us and Dr David Wakefield for insightful suggestions. Mr Danny Ball, Columbia Scientific Balloon Facility and Mr Loren Seely, Aerostar International have provided advice and materials for this study. Financial support from the NASA Balloon Program Office is gratefully acknowledged.

\section{References}

Haj-Ali, R.M. and Muliana, A.H. (2004). Numerical finite element formulation of the Schapery nonlinear viscoelastic material model. International Journal for Numerical Methods in Engineering, 59, 25-45. 
Henderson, J.K., Caldron, G. and Rand, J.L. (1994). A nonlinear viscoelastic constitutive model for balloon films. American Institute of Aeronautics and Astronautics AIAA-1994-638.

ABAQUS (2003). ABAQUS Version 6.3. ABAQUS, INC. 1080 Main Street Pawtucket, Rhode Island 02860-4847 USA, Pawtucket, RI 02860.

McCrum, N.G., Buckley, C.P. and Bucknall, C.B. (2003). Principles of Polymer Engineering, 2nd edition, Oxford Science Publications, Oxford.

Pagitz, M. and Pellegrino, S. (2007). Buckling pressure of "pumpkin" balloons. Accepted for publication in International Journal of Solids and Structures.

Photomodeler (2004). Pro5.0 EOS Systems.

Rand, J.L., Grant, D.A. and Strganac, T. (1996) The nonlinear biaxial characterization of balloon film. American Institute of Aeronautics and Astronautics AIAA-96-0574.

Rand, J.L., Henderson, J.K. and Grant, D.A. (1996). Nonlinear behaviour of linear low-density polyethylene. Polymer Engineering and Science, 36 (8), 1058-1064.

Schapery, R.A. (1969). On the characterization of nonlinear viscoelastic materials. Polymer Engineering and Science, 9 (4), 295-310.

Smith, M.S. and Rainwater, E.L. (2004). Optimum designs for superpressure balloons. Advances in Space Research, 33, 1688-1693.

Vialettes, P., Siguier, J-M., Guigue, P., Karama, M., Mistou, S., Dalverny, O., and Petitjean, F. (2005). Viscoelastic laws study for mechanical modelization of super-pressure balloons. American Institute of Aeronautics and Astronautics AIAA-2005-7469. 